\title{
Effects of Phase IV Pedometer Feedback Home-Based Cardiac Rehabilitation on Cardiovascular Functional Capacity in Patients With Myocardial Infarction: A Randomized Controlled Trial
}

\author{
Mostafa Dehghani ${ }^{1,2}$, Mostafa Cheraghi ${ }^{3,2}$, Mehrdad Namdari ${ }^{3,2}$, Valiollah Dabidi Roshan ${ }^{4 *}$ \\ ${ }^{1} \mathrm{PhD}$ cardiovascular and Respiratory physiology, University of Mazandaran, Babolsar, Iran \\ ${ }^{2}$ Cardiovascular Research Center, Shahid Rahimi Hospital, Lorestan university of Medical Science, Khorramabad, Iran \\ ${ }^{3}$ Department of Cardiology, Lorestan University of Medical Sciences, Khorramabad, Iran \\ ${ }^{4}$ Department of Sport Physiology, College of Physical Education and Sport Sciences, University of Mazandaran, Babolsar, Iran
}

*Correspondence to Valiollah Dabidi Roshan Tel: +989113151509

Email:vdabidiroshan@yahoo.com

Received November 29, 2018 Accepted January 12, 2019 Published online June 30, 2019
Please cite this article as follows: Dehghani $M$, Cheraghi M, Namdari $M$ Dabidi Roshan V. Effects of phase IV pedometer feedback home-based cardiac rehabilitation on cardiovascular functional capacity in patients with myocardial infarction: a randomized controlled trial. Int J Basic Sci Med. 2019;4(2):7580. doi:10.15171/ ijbms.2019.15.

\begin{abstract}
Introduction: Pedometer feedback home-based cardiac rehabilitation (PFHCR) programs have been effective in augmenting exercise tolerance. Our aim was to investigate the effects of PFHCR on cardiovascular functional capacity in patients with myocardial infarction (MI).

Methods: Forty MI patients were divided into two intervention and two control groups $(n=10)$ in a randomized controlled trial. The intervention group received a PFHCR program including routine medications along with continuous exercise program, whereas the control group received traditional care without PFHCR. Baseline evaluations and cardiovascular stresses were controlled during the 8-week follow-up rehabilitation program in all patients. Data analysis was conducted using one-way ANOVA and paired sample student's t-test $(P \leq 0.05)$.

Results: At baseline, no significant differences were observed between the groups. After eight weeks of PFHCR, the intervention groups had significantly higher metabolic equivalent $(P=0.001)$, VO2max $(P=0.001)$, total exercise times $(P=0.001)$, and total distance traveled $(P=0.003)$ when compared with the control groups. However, no significant intragroup or intergroup differences in variables were observed between the men and women.

Conclusion: Our results showed that PFHCR exhibited significant optimal effects on the cardiovascular functional capacity in MI patients.

Keywords: Cardiac rehabilitation, Myocardial infarction, Functional capacity, Exercise test, Coronary heart disease
\end{abstract}

\section{Introduction}

Cardiovascular diseases (CVDs) includes coronary heart disease (CHD), congestive heart failure, myocardial infarction (MI), peripheral artery disease, and carotid artery disease. Coronary artery disease (CAD), angina pectoris, and MI are among the main causes of death worldwide. According to the World Health Organization (WHO), mortalities due to CAD will increase from 7.1 million in 2002 to 11.1 million in $2020 .^{1,2}$ Evidence has shown that only one-third of all MI cases lead to serious heart diseases and death. ${ }^{3}$ Hence, post-MI therapies have become the main focus of studies in medicine and sport science. Although the effect of regular aerobic exercises has been clarified on early prevention of cardiovascular diseases and risk of death caused by them, ${ }^{4,5}$ supervised physical activity (PA) can prevent the relapse of cardiovascular events. Cardiac rehabilitation (CR) home-based programs are more effective than $\mathrm{CR}$ methods in hospitals and health centers due to needing less time and expense to perform, ${ }^{6}$ reducing the likelihood of readmission ${ }^{7}$ and risk of recurrent $\mathrm{MI},{ }^{5}$ and upgrading physical performance. $^{3}$ However, some patients do not perform adequate levels of PA to experience its health benefits. ${ }^{8}$

Research evidence shows that walking

(c) 2019 The Author(s); Published by Zabol University of Medical Sciences. This is an open-access article distributed under the terms of the Creative Commons Attribution License (http://creativecommons.org/licenses/by/4.0), which permits unrestricted use, distribution, and reproduction in any medium, provided the original work is properly cited. 
is a simple activity for cardiovascular patients and a flexible alternative for people who do not have access to hospital treatments. In addition, these types of therapeutic approaches play a major role in resuming everyday work and life in such patients. ${ }^{9}$ Parmanto and Saptono ${ }^{10}$ reported that 150 minutes of walking per week was enough to achieve health objectives, but TudorLocke et al stated that walking 100 steps per minute was an activity of moderate intensity and walking 3000 steps per week could have cardiovascular benefits. ${ }^{11}$ Currently, it is not clear whether exercise approaches that include conventional programs (PA recommendations without a pedometer feedback) or pedometer-based programs will have benefits for MI patients.

Research findings indicate that only $10-20 \%$ of patients with acute MI participate in a secondary preventive program based on $\mathrm{PA}^{3}$ and only $40 \%$ of patients taking part in the maintenance phase (Phase IV) of CR programs manage to achieve the recommended PA level. ${ }^{12} \mathrm{CR}$ programs are employed to recover health and prevent disease progression in people with heart diseases ${ }^{3}$ and play a major role in secondary prevention (SP) ${ }^{12}$ However, patients with MI do not usually perform adequate postoperative PA to prevent SP due to fear of movement and muscle weakness caused by immobility.

Hence, the present study investigated the assumption that use of a pedometer can increase daily home-based CR by creating self-motivation and thus is able to improve cardiovascular function and physical performance capacity in MI patients. Accordingly, the present study aimed to study the effects of pedometer-based feedback in an 8-week pedometer feedback home-based CR (PFHCR) program on cardiac functional parameters [i.e. metabolic equivalents (METs), VO2max, total exercise times following an aerobic exhaustive exercise on treadmill, distance traveled, and maximum heart rate during the Bruce test] in men and women with $\mathrm{MI}$ in comparison to those of a conventional treatment program.

\section{Methods}

\section{Participants}

The study population consisted of all MI patients hospitalized in the cardiacintensivecare unitand cardiology ward of Shahid Madani hospital, Khoramabad, Iran from September to November 2017. Based on diagnosis made by specialists and the New York Heart Association (NYHA) classification, ${ }^{13}$ all potential participants were diagnosed with heart failure and were classified as NYHA class II and III. They had left ventricular ejection fraction of $40-55 \%$ in electrocardiography, and took part in the outpatient program after discharge. Following MI, the patients had no persistent complications and were in phase IV CR. Ninety-five patients were initially enrolled (Figure 1), 55 of whom fulfilled the inclusion criteria. Finally, 44 male and female patients with MI aged 45-60 volunteered to participate in the study. They were randomly divided into four groups: 2 intervention groups (10 males and 10 females) and 2 control groups (12 men and 12 women). The intervention groups underwent $\mathrm{CR}$ walking program using waist-mounted pedometers (PA-S20, Switzerland) according to the manufacturer's instructions, and the number of steps taken, shown by the step counter, were recorded in a sport results notebook. The control groups performed the conventional CR program at 5:00 PM without pedometer feedback. Participants in the control group also received traditional care but were given no exercise education. The participants were asked to return after eight weeks for evaluations. After baseline evaluations, participants were randomly allocated to the intervention groups (with pedometer) or control groups (without pedometer).

\section{Inclusion and Exclusion Criteria}

The inclusion criteria were history of angioplasty, CAD and/or angiography of moderate CVD (e.g. mild CAD), lack of taking beta blockers (propranolol, methoral [metoprolol], verapamil, atenolol, and carvedilol) 24 hours before the exercise test, having physical ability to get the exercise test (e.g. lack of suffering from arthritis or amputation), lack of smoking, lack of drinking alcohol, and lack of taking antioxidant supplements, lack of having ECG abnormalities (e.g. signs of heart failure), and lack of suffering from infectious diseases or cold (because of the likelihood of abnormal ECG manifestations). The exclusion criteria were definite diagnosis of ischemia, lack of being permitted to get the exercise test, having ejection fraction of less than $40 \%$ and over $55 \%$, and lack of history of using pacemaker (for CVD patients).

\section{Interventional training}

The PFHCR program in this study included walking at home for almost 45-60 minutes (7-minute warm-up, 40-minute walking, and 7-minute recovery and stretching exercises) and at 11-13 Borg score of perceived exertion. Five weekly exercise sessions were conducted in the afternoon for eight weeks with and without step counter feedback for both women and men in the intervention and control groups, respectively. The 8-week walking exercise was designed based on a protocol used in a previous study ${ }^{14}$ yet with minor modifications. From the second week to the eighth week, the number of steps increased by $10 \%$ per week (by 100 steps/day to a total of 500 steps/week). In other words, 100 steps were added every day, as the extra load, to the number of steps taken. The participants were trained to add the extra load during the same 40 minutes of daily walking. A pilot study was conducted to evaluate the way according to which the progressive protocol was to be carried out by the participants and the number of steps to be taken at the start (3,500 steps/day). The control groups performed the same CR exercise program but did not use the step counter feedback. 


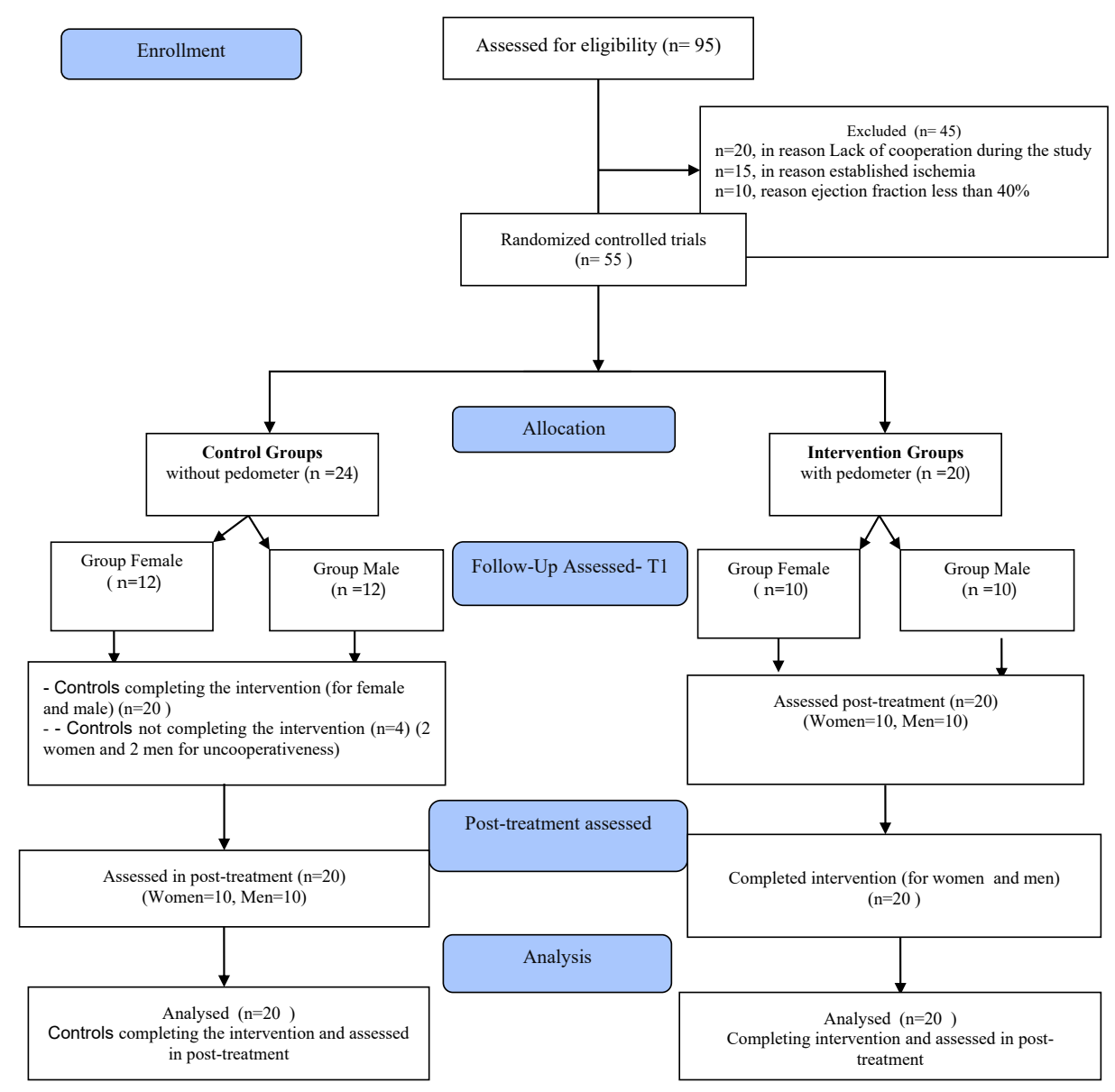

Figure 1. CONSORT Flowchart of the Trial.

The Bruce Protocol Stress Test and Assessment of Cardiovascular Functional Capacity Indicators and Termination Criteria

The protocol was implemented as a two-stage project (before and after the 8-week exercise-based CR). In both stages, the advanced treadmill test was performed using the modified Bruce Protocol (at $3 \mathrm{~km} / \mathrm{h}$ rate with increases of $1.4 \mathrm{~km} / \mathrm{h}$ after 3 minutes, followed by a $3 \%$ increase in grade at a constant speed) to determine the cardiovascular and respiratory function indicators of aerobic exhaustive exercise.${ }^{15}$ Functional capacity including metabolic equivalent of task (MET), VO2max, total exercise times following an aerobic exhaustive exercise on the treadmill and the distance traveled on the treadmill during the activity were also measured and recorded. Before implementing the standardized Exercise Treadmill Test (ETT) using the Bruce Protocol to determine cardiac and respiratory function, the patients were examined by the physician in attendance, and were asked to wear light clothing and take off their shoes to measure their height and weight using standard methods. The exercise termination criteria, previously described, ${ }^{16}$ were assessed during the last minute of each stage of the test.

\section{Statistical Analysis}

The SPSS version 22 (IBM Inc., New York, US) was used to perform statistical analysis. Normal distribution of data was investigated using the Shapiro-Wilk test. $\mathrm{P}<0.05$ was considered significance level. Data was analyzed using one-way ANOVA and paired sample Student's t-test to investigate intragroup and intergroup differences in the studied variables.

\section{Results}

All demographic and clinical characteristics of the participants and clinical variables during hospitalization including age, gender, CHD classification, disease intensity, waist circumference, BMI, blood pressure, medications, and smoking were studied (Table 1). A total of $40 \mathrm{MI}$ patients were evaluated during the 8 weeks. They were randomly divided into two intervention groups and two control groups $(\mathrm{N}=10)$. Table 1 presents baseline, demographic, and clinical characteristics of our patients. There was no significant difference in CHD classification, age, BMI, addiction, high blood pressure, family history of heart diseases or diabetes, coronary artery bypass grafting, ST-segment elevation myocardial infarction (STEMI), and non-STEMI between the groups. 
Table 1. Baseline Characteristics of Participants

\begin{tabular}{|c|c|c|c|c|c|c|}
\hline \multirow[b]{2}{*}{ Variables } & \multicolumn{3}{|l|}{ Male $(n=20)$} & \multicolumn{3}{|l|}{ Female $(n=20)$} \\
\hline & $\begin{array}{l}\text { Intervention Group } \\
\mathbf{n}=\mathbf{1 0}\end{array}$ & $\begin{array}{l}\text { Control Group } \\
n=10\end{array}$ & $P$ value & $\begin{array}{l}\text { Intervention Group } \\
\mathrm{n}=10\end{array}$ & $\begin{array}{l}\text { Control Group } \\
\mathrm{n}=10\end{array}$ & $P$ value \\
\hline Age $(y)$, mean $\pm S D$ & $51.4 \pm 7.97$ & $51.1 \pm 7.86$ & 0.559 & $51.5 \pm 6.96$ & $53 \pm 7.33$ & 0.331 \\
\hline $\mathrm{BMI}\left(\mathrm{kg} / \mathrm{m}^{2}\right)$, mean $\pm \mathrm{SD}$ & $23.66 \pm 1.58$ & $24.6 \pm 1.20$ & 0.442 & $24.53 \pm 1.28$ & $25.9 \pm 1.43$ & 0.124 \\
\hline Smoking, No. (\%) & $2(20)$ & $2(20)$ & 0.653 & - & - & 0.411 \\
\hline Hypertension, No. (\%) & $3(30)$ & $3(40)$ & 0.371 & $3(30)$ & $2(20)$ & 0.204 \\
\hline Family history of heart disease, No. (\%) & $3(30)$ & $3(30)$ & 0.211 & $3(30)$ & $2(20)$ & 0.131 \\
\hline Diabetes mellitus, No. (\%) & $1(10)$ & $1(10)$ & 0.756 & $2(20)$ & $2(20)$ & 0.698 \\
\hline CABG, No. $(\%)$ & $3(30)$ & $2(20)$ & 0.151 & $2(20)$ & $2(20)$ & 0.254 \\
\hline NSTEMI, No. (\%) & $1(10)$ & $2(20)$ & 0.134 & $2(20)$ & $2(20)$ & 0.372 \\
\hline STEMI, No. (\%) & $1(10)$ & $1(10)$ & 0.347 & $2(20)$ & $2(20)$ & 0.267 \\
\hline
\end{tabular}

CABG: coronary artery bypass grafting; NSTEMI: non-ST segment elevation myocardial infarction; STEMI: ST-segment elevation myocardial infarction.

* Significant difference at $P \leq 0.05$.

Assessment of Functional Capacity in the Exercise Test The effects of the exercise test on cardiovascular performance in participants are presented in Table 2 . In the baseline evaluation, there were no statistically significant differences in any of the variables between the control groups. With regards to intragroup and intergroup effects after receiving the PFHCR, significant differences were observed in MET, VO2max, total exercise times and distance traveled during Bruce test between the female and male participants in the intervention group compared to the control group following the 8-week CR. However, no significant intragroup differences were observed between men and women in control groups.

\section{Discussion}

Recent studies on patients participating in CR have programs revealed that many of them do not meet the recommendations for physical activity. ${ }^{17}$ This indicates the need for pedometer feedback during rehabilitation programs at home and in real life settings more than ever. Based on our information, few studies have so far evaluated the effects of PFHCR on VO2max based on estimated functional capacity, MET, total exercise time, and distance traveled in MI patients. Our results showed that PFHCR influenced these indicators in both female and male MI patients compared to the control groups.

MET that is a standardized measure of energy consumption is strongly associated with increased risk of CHD, adverse cardiovascular outcomes, and allcause and cardiovascular mortality. In the Scandinavian Simuvastatin Survival Study ${ }^{18}$ MET was commonly detected in patients with CHD. We in this study used MET to detect overall metabolic changes and to determine the impacts of our intervention. In our study, MET significantly improved in intervention group after 8-week follow-up. The modified Vo2max, total exercise times and distance traveled significantly improved in the intervention group. Therefore, the PFHCR was effective to improve cardiac function in MI patients.

CR should be considered as an integral component of any cardiology prevention-service continuum. ${ }^{18}$ In patients with heart failure, VO2max is reduced because the respiratory restriction prevents the maximum capacity of the heart. Additionally, higher exercise intensity had greater impact on VO2max. ${ }^{19,20}$ A significant difference was observed in $\mathrm{VO} 2 \mathrm{max}$ compared to baseline between

Table 2. Comparison of Cardiac Functional Parameters Before and After Cardiac Rehabilitation Between Females and Males in the Control and Intervention Groups

\begin{tabular}{|c|c|c|c|c|c|}
\hline \multirow{2}{*}{ Functional capacity parameter } & & \multicolumn{2}{|c|}{ Control Groups } & \multicolumn{2}{|c|}{ Intervention Groups } \\
\hline & & Pre & Post & Pre & Post \\
\hline \multirow{2}{*}{$\begin{array}{l}\text { Metabolic equivalent of task } \\
(\mathrm{MET})(\mathrm{O} 2)\end{array}$} & Female & $8.08 \pm 2.98$ & $7.45 \pm 2.38$ & $8.14 \pm 1.6$ & $10.97 \pm 2.13^{* * *}$ \\
\hline & Male & $10.08 \pm 3.1$ & $9.77 \pm 3.26$ & $8.23 \pm 2.49$ & $11.29 \pm 2.21^{* * *}$ \\
\hline \multirow{2}{*}{ VO2max (mL/kg/min) } & Female & $26.12 \pm 8.34$ & $29.13 \pm 9.65$ & $28.52 \pm 5.57$ & $38.40 \pm 7.47^{* * *}$ \\
\hline & Male & $34.48 \pm 8.99$ & $33.40 \pm 9.55$ & $28.83 \pm 8.72$ & $39.52 \pm 7.74^{* * *}$ \\
\hline \multirow{2}{*}{ Total exercise times (min) } & Female & $5.46 \pm 2.78$ & $5.32 \pm 2.41$ & $5.33 \pm 1.94$ & $8.01 \pm 2.40^{* * *}$ \\
\hline & Male & $6.79 \pm 2.63$ & $7.32 \pm 2.35$ & $5.91 \pm 1.66$ & $7.99 \pm 2.15^{* * *}$ \\
\hline \multirow{2}{*}{ Distance traveled $(\mathrm{M})$} & Female & $340 \pm 21$ & $290.10 \pm 18$ & $308.20 \pm 14$ & $511.50 \pm 26^{* * *}$ \\
\hline & Male & $447.90 \pm 24$ & $480.70 \pm 24$ & $365.10 \pm 13$ & $557.80 \pm 20^{* *}$ \\
\hline
\end{tabular}

Values expressed as mean \pm SD.

* Significant difference at $P \leq 0.05$. ${ }^{*} P<0.05,{ }^{*} P<0.01, * * * P<0.001$. 
the studied groups in our study. However, PFHCR intervention significantly improved $\mathrm{VO} 2 \mathrm{max}$ after 8 weeks compared to the traditional care.

Epidemiological studies indicate that even a slight increase in distance traveled per daily is effective to improve heart health. Based on these findings, general practitioners and health professionals play a key role in encouraging patients to increase distance traveled per day. As a preventive method, physical activity significantly reduces cardiovascular disease mortality by moderating risk factors such as increased blood flow in coronary artery branches and increased cardiac and muscle oxygen consumption. ${ }^{21}$ It has been confirmed that $\mathrm{VO} 2 \mathrm{max}$ is increases following regular physical exercises because of enhanced mitochondrial enzymes activity and increased myoglobin levels and skeletal muscles vasculature. ${ }^{22}$ In the posttest of the present study, $\mathrm{VO} 2 \mathrm{max}$ and METS values also significantly increased in men and women in the intervention group but decreased in men and women in the control group. The changes in the intervention group were also significant compared to the control group. Moreover, changes in VO2max and MET in both intervention groups and in both genders were in agreement with changes in the time to exhaustion during treadmill running and distance traveled shown by the pedometers. The present study showed that VO2max in PFHCR increased in men and women in intervention groups $(P<0.001)$ after the 8 -week $C R$ program but no significant corresponding differences were observed in the control groups $(P \geq 0.05)$.

Aerobic exercise increases functional capacity, and 1 unit increase in functional capacity reduces the risk of cardiovascular events by $25 \% .^{23,24}$ It has been confirmed that $\mathrm{VO} 2 \mathrm{max}$ increases following regular physical exercises because of enhanced activity of mitochondrial enzymes, increased myoglobin levels, and augmented capillary surfaces in the skeletal muscles. ${ }^{25}$ Our results showed significant increase in $\operatorname{MET}(P<0.001)$ in male and female MI patients after the 8 -week PFHCR program. Besides, PFHCR results in a significant increase in aerobic capacity in individuals with sedentary lifestyle. The increase of functional capacity in male and female participants in intervention groups in the present research was accompanied by improved cardiac functional capacity in female and male participants in PFHCR groups compared to the control group $(P \leq 0.05)$. Sharma and McLeod noticed that CR significantly affected physical tolerance of patients. ${ }^{26}$ In a study on 49 patients with MI after CABG procedure, Park et al observed that their functional capacity clearly improved after CR. ${ }^{27}$ Given their findings, the increased walking stamina of women and men in PFHCR groups in the present study may also be due to improved function of the respiratory and cardiovascular systems that decreased the patients' locomotor disability. The small number of participants in the intervention groups was one of the limitations of our study. Future studies can obtain more reliable results if they are conducted with larger sample size and more careful control of patients' activities at home.

\section{Conclusions}

The present study showed improved cardiac functional parameters following the 8-week PFHCR program in MI patients. These changes including improved myocardial workload and oxygen consumption as well as improved indicators of cardiovascular function were gender independent and equal in men and women. Therefore, pedometers can be used for patients that do not have access to CR centers, cannot go to sports facilities for financial or otherwise reasons, or lack free time for walking.

\section{Ethical Approval}

All participants provided written informed consent to participate in the study and the study protocol conformed to the Declaration of Helsinki and was approved by the local ethics committee (Approval ID: IR.LUMS.REC.1397.105). All procedure of the study was registered in the Iranian Registry of Clinical Trials (identifier: IRCT20181122041725N1; https://www.irct. ir/trial/35414).

\section{Competing interest}

The authors declare no competing interests.

\section{Acknowledgements}

This study was supported by the Mazandaran and Lorestan Universities of Medical Sciences. The authors would like to express their gratitude to the Specialized Heart Health Center at Cardiovascular Research Center of Shahid Rahimi Hospital and to all the patients who did their best in cooperating with the study.

\section{References}

1. Gupta R, Joshi P, Mohan V, Reddy KS, Yusuf S. Epidemiology and causation of coronary heart disease and stroke in India. Heart. 2008;94(1):16-26. doi:10.1136/hrt.2007.132951

2. Abdollahi AA, Hoseini SA, Salehi A, Behnampour N, Abasi A. Coronary artery lesions and some of its related factors in more than 5000 patients in kosar Angiography Center (Golestan Province) from 2007 to 2009. Scientific Journal of Kurdistan University of Medical Sciences. 2012;17(1):18-24. [Persian].

3. Morrow DA, Gersh BJ. Chronic coronary artery disease. In: Libby P, Bonow RO, Mann DL, Zipes DP, Braunwald E, eds. Braunwald's heart disease: A textbook of cardiovascular medicine. 8th ed. Philadelphia: Elsevier Saunders; 2008:13531417. doi:10.1007/978-1-4419-1005-9_1256

4. Garza MA, Wason EA, Zhang JQ. Cardiac remodeling and physical training post myocardial infarction. World J Cardiol. 2015;7(2):52-64. doi:10.4330/wjc.v7.i2.52

5. Black DR, Coster DC, Paige SR. Physiological health parameters among college students to promote chronic 
disease prevention and health promotion. Prev Med Rep. 2017;7:64-73. doi:10.1016/j.pmedr.2017.05.006

6. O'Malley G, Ring-Dimitriou S, Nowicka P, et al. Physical activity and physical fitness in pediatric obesity: what are the first steps for clinicians? Expert conclusion from the 2016 ECOG workshop. Int J Exerc Sci. 2017;10(4):487-496.

7. Anderson L, Sharp GA, Norton RJ, et al. Home-based versus centre-based cardiac rehabilitation. Cochrane Database Syst Rev. 2017;6:Cd007130. doi:10.1002/14651858.CD007130. pub4

8. Anderson L, Thompson DR, Oldridge N, et al. Exercise-based cardiac rehabilitation for coronary heart disease. Cochrane Database Syst Rev. 2016(1):Cd001800. doi:10.1002/14651858. CD001800.pub3

9. Ter Hoeve N, Sunamura M, Stam HJ, et al. Effects of two behavioral cardiac rehabilitation interventions on physical activity: A randomized controlled trial. Int J Cardiol. 2018;255:221-228. doi:10.1016/j.ijcard.2017.12.015

10. Brennan DM, Tindall L, Theodoros D, et al. A blueprint for telerehabilitation guidelines--October 2010. Telemed J E Health. 2011;17(8):662-665. doi:10.1089/tmj.2011.0036

11. Parmanto B, Saptono A. Telerehabilitation: State-of-theArt from an Informatics Perspective. Int $\mathrm{J}$ Telerehabil. 2009;1(1):73-84.

12. Tudor-Locke C, Craig CL, Aoyagi Y, et al. How many steps/ day are enough? For older adults and special populations. Int $J$ Behav Nutr Phys Act. 2011;8:80. doi:10.1186/1479-5868-8-80

13. Dolgin M, Fox AC, Gorlin R, Levin RI, New York Heart Association. Criteria Committee. Nomenclature and criteria for diagnosis of diseases of the heart and great vessels. 9th ed. Boston, MA: Lippincott Williams \& Wilkins; 1994.

14. Kaminsky LA, Jones J, Riggin K, Strath SJ. A pedometerbased physical activity intervention for patients entering a maintenance cardiac rehabilitation program: a pilot study. Cardiovasc Diagn Ther. 2013;3(2):73-79. doi:10.3978/j. issn.2223-3652.2013.03.03

15. Fornitano LD, Godoy MF. [Increased rate-pressure product as predictor for the absence of significant obstructive coronary artery disease in patients with positive exercise test]. Arq Bras Cardiol. 2006;86(2):138-144. doi:10.1590/s0066$782 \times 2006000200010$

16. Dimeo F, Pagonas N, Seibert F, Arndt R, Zidek W, Westhoff TH. Aerobic exercise reduces blood pressure in resistant hypertension. Hypertension. 2012;60(3):653-658. doi:10.1161/ hypertensionaha.112.197780

17. Pinkstaff S, Peberdy MA, Kontos MC, Finucane S, Arena R. Quantifying exertion level during exercise stress testing using percentage of age-predicted maximal heart rate, rate pressure product, and perceived exertion. Mayo Clin Proc. 2010;85(12):1095-1100. doi:10.4065/mcp.2010.0357

18. Girman CJ, Rhodes T, Mercuri M, et al. The metabolic syndrome and risk of major coronary events in the Scandinavian Simvastatin Survival Study (4S) and the Air
Force/Texas Coronary Atherosclerosis Prevention Study (AFCAPS/TexCAPS). Am J Cardiol. 2004;93(2):136-141. doi:10.1016/j.amjcard.2003.09.028

19. Oldridge N. Exercise-based cardiac rehabilitation in patients with coronary heart disease: meta-analysis outcomes revisited. Future Cardiol. 2012;8(5):729-751. doi:10.2217/fca.12.34

20. Achttien RJ, Staal JB, van der Voort S, et al. Exercise-based cardiac rehabilitation in patients with coronary heart disease: a practice guideline. Neth Heart J. 2013;21(10):429-438. doi:10.1007/s12471-013-0467-y

21. Bravata DM, Smith-Spangler C, Sundaram V, et al. Using pedometers to increase physical activity and improve health: a systematic review. JAMA. 2007;298(19):2296-2304. doi:10.1001/jama.298.19.2296

22. Riahi S, Mohammadi MT, Sobhani V, Ababzadeh S. Chronic aerobic exercise decreases lectin-like low density lipoprotein (lox-1) receptor expression in heart of diabetic rat. Iran Biomed J. 2016;20(1):26-32. doi:10.7508/ibj.2016.01.004

23. Bairey Merz CN, Alberts MJ, Balady GJ, et al. ACCF/AHA/ ACP 2009 competence and training statement: a curriculum on prevention of cardiovascular disease: a report of the American College of Cardiology Foundation/American Heart Association/American College of Physicians Task Force on Competence and Training (Writing Committee to Develop a Competence and Training Statement on Prevention of Cardiovascular Disease): developed in collaboration with the American Academy of Neurology; American Association of Cardiovascular and Pulmonary Rehabilitation; American College of Preventive Medicine; American College of Sports Medicine; American Diabetes Association; American Society of Hypertension; Association of Black Cardiologists; Centers for Disease Control and Prevention; National Heart, Lung, and Blood Institute; National Lipid Association; and Preventive Cardiovascular Nurses Association. Circulation. 2009;120(13):e100-126. doi:10.1161/circulationaha.109.192640

24. Sandor B, Nagy A, Toth A, et al. Effects of moderate aerobic exercise training on hemorheological and laboratory parameters in ischemic heart disease patients. PLoS One. 2014;9(10):e110751. doi:10.1371/journal.pone.0110751

25. Kraus WE, Houmard JA, Duscha BD, et al. Effects of the amount and intensity of exercise on plasma lipoproteins. N Engl J Med. 2002;347(19):1483-1492. doi:10.1056/ NEJMoa020194

26. Sharma R, McLeod AA. Cardiac rehabilitation after coronary artery bypass graft surgery: its effect on ischaemia, functional capacity, and a multivariate index of prognosis. Coronary Health Care. 2001;5(4):189-193. doi:10.1054/chec.2001.0142

27. Perk J, Hedbäck B, Engvall J. Effects of cardiac rehabilitation after coronary artery bypass grafting on readmissions, return to work, and physical fitness. A case-control study. Scand J Soc Med. 1990;18(1):45-51. 